\title{
Spontaneous shears in smectic elastomers
}

\author{
J. M. Adams and M. Warner \\ Cavendish Laboratory, University of Cambridge, Madingley Road, Cambridge CB3 OHE, United Kingdom
}

(Received 8 August 2005; published 22 March 2006)

\begin{abstract}
We model the spontaneous elastic shear deformations of smectic elastomers as they make the thermal transition between the $A$ and $C$ phases. Tilt of the director is accompanied by shear. Unlike nematic elastomers where spontaneous elongation is associated with changes in the magnitude of order, spontaneous distortion in smectics is instead shear and is associated with rotation of the director of the underlying nematic order. The effect of the multistage cross-linking associated with creating monodomain elastomers is taken into account. The angle of molecular tilt and the angle associated with spontaneous shear deformation are not simply related.

DOI: 10.1103/PhysRevE.73.031706

PACS number(s): 61.30.Vx, 83.80.Va, 83.80.Xz, 62.20.Dc
\end{abstract}

\section{INTRODUCTION}

Liquid crystalline elastomers display macroscopic mechanical phenomena that arise from the coupling of their liquid crystalline order with the elastic structural elements. Nematic order is orientational and causes elongation to a prolate chain shape, or more rarely, flattening to oblate. As the order changes, so too there are spontaneous (that is, not externally imposed) mechanical strains that reflect the coupling between orientation and shape. These strains can be very large, a sample elongating by as much as factors of 4-7. Temperature change or illumination can change the order, thus giving rise to thermal or optical actuation. Spontaneous shape change is an important measure of the anisotropy achieved and determines directly the range of strains over which soft elastic response is achieved. This dramatic form of elasticity obtains in systems with a responsive underlying order [1]. Some shape changes can be imposed in ideal systems without energy cost-certain elastic moduli vanish and there is an associated absence of restoring forces along some symmetry directions. In nematic elastomers the underlying order is orientation with a director $\mathbf{n}$ defining its direction. The soft elasticity occurs when strain is imposed in a plane containing the director which responds by rotating. It takes with it the anisotropic distribution of chain shapes at constant polymer entropy and nematic order [2]. The chains are accommodated by macroscopic shape change that is therefore at constant free energy because of the constancy of entropy and nematic energy $[3,4]$. Nonideality in the network leads in practice to a small energy cost, semisoftness, to which we shall refer in this paper. The relation between the amplitude of soft deformation and the extent of spontaneous deformation, and their correspondence to nematic elastomer experiments, is discussed in some detail [2]. A primary motivation of the present study of spontaneous deformation is to make contact with theoretical work on smectic $C$ soft elasticity $[5,6]$ where the amplitude of the soft deformations associated with this symmetry-breaking process is twice the extent of the spontaneous shear.

Spontaneous shape change in nematic elastomers arises from a change in the magnitude of the orientational order. We wish to investigate shape change in very different elastomers (smectics) where instead spontaneous rotations of the director of the underlying nematic order are important, namely in cooling further from the transition $A$ to $C$. The $A$ phase has the underlying nematic (uniaxial, orientational) order directed along the layer normal. At the $A C$ transition the director starts tilting by an angle $\theta$ with respect to the layer normal that grows continuously from zero. For the unwound $\mathrm{SmC}$ phase, one expects $[7,5]$ associated spontaneous inplane shears to develop.

The first $\mathrm{SmC}$ elastomer monodomains [8,9] resulted from cross-linking a $\mathrm{Sm} C^{*}$ melt oriented by the electric field of two enclosing glass electrodes. Subsequent mechanical deformation, for instance, in response to reversal of the electric field, was necessarily constrained by these rigid electrodes and many interesting phenomena were thereby masked. An example is the switching of ferroelectric polarization which is accompanied by mechanical shears-when the latter are suppressed a much larger barrier to polarization reversal is created.

Another strategy is to apply mechanical fields to large, free-standing strips of elastomer. For instance, one first partially cross-links the elastomer and then elongates the strip to uniformly align the director, but leaving the planes in polydomains with their normal randomly arranged on a cone about the stretch direction. Then, after a second crosslinking, one imposes another elongation at an angle to the first extension equal to the tilt angle of the $\mathrm{SmC}$ phase. The second stretch makes the tilt direction uniform [10]. Annealing and cooling before releasing the extension then made the monodomain stable without another cross-linking step. Naturally such monodomains could not be examined for their spontaneous deformations as temperature was elevated since weakening the smectic order caused a reversion to the polydomain structure.

Later, the Finkelmann multi-stage cross-linking technique of obtaining single liquid crystal elastomers, but using inplane shear followed by another cross-linking step, was applied [11] to obtain $\mathrm{SmC}$ monodomains. More recently the same technique has been applied [12] to $\operatorname{Sm} A$ elastomers displaying a lower temperature $C$ phase. Very large spontaneous shears, of the order of 0.5 , have been observed on cooling. The shear has an offset, i.e., even while in the $A$ phase the sample remained sheared. In this work we associate the shear off-set with the two-step cross-linking process of making a monodomain. The angle of molecular tilt is not simply related to the angle associated with shear. It was rec- 
ognized early [7] that the two angles could not simply be identified. That analysis, including nonlinear effects at low order, found for small tilts the tangent of the spontaneous shear angle equal to the tilt angle times the ratio of two elastic constants. A nonsimple connection also arises in a nonlinear, Lagrangian elastic analysis [5] of the spontaneous shears arising at and below the $A$ to $C$ smectic transition. We shall give explicit results for this connection up to large tilts deep into the nonlinear regime now explored by experiment. Our purpose here is to model the significant results of spontaneous and residual shears seen in the experiments of [12].

The layer spacing in smectics, liquid or elastomeric, is very rigid compared with the underlying rubbery matrix. It is often the case [13] in smectic elastomers that chain crosslinks are localized to remain in a layer close to where the link was formed. Thereafter deformations of the rubber matrix are not independent of the smectic layer structure. Displacements of the rubber must convect the layers with it. Necessarily the rubber can then only deform by respecting the strong layer constraint [14]. (This coupling is the opposite of the case of smectic liquids in aerogels.) Thus these smectic elastomers are in effect two-dimensional rubbers-most extensions and shears are rubberlike, but extensions along the layer normal sense the smectic order and the modulus rises by two orders of magnitude [13]. We shall consider elastomers where the layer spacing constraint is very strong and tightly restricts the class of allowed elastic deformations, including spontaneous deformations, to those that respect the equilibrium layer spacing. Fully nonlinear elasticity theory, valid up to the large strains possible in elastomers and respecting layering and constancy of volume, have recently been developed [15] and model the remarkable rubber elasticity that arises.

\section{RELAXATION AFTER MULTISTAGE CROSS- LINKING: SPONTANEOUS DEFORMATIONS ON $A$ TO $C$ TRANSITIONS}

The model of $\operatorname{Sm} A$ and $\operatorname{Sm} C$ elastomers we will use is derived for $\operatorname{Sm} A$ elastomers in [15] and shows close agreement with experiment [13]. As in earlier continuum models $[7,16]$, the underlying network is assumed to be essentially nematic. Its essential elastic response is rubbery whenever layer spacing is not being altered. For an adequate description of the elastic response, we thus require the nematic elastomer free energy, but with variations of sample shape constrained by the rigidity of the smectic order.

Monodomain samples are essential in obtaining spontaneous distortions on thermally inducing order change. Nematic monodomain elastomers can be obtained by cross-linking chains nematically ordered in a uniaxial field or by exploiting surface alignment in thin enough samples. However, the most common technique is that of Finkelmann [17] where a sample is partially cross-linked so that it is solid enough to hold and thus uniaxially stretch, whereupon cross-linking is then completed. On release, the sample partially retracts to a new stress-free, equilibrium shape. It is on this relaxed state that mechanical experiments are performed. Strains, imposed or spontaneous because of further temperature change, are measured with respect to this state. Stresses must be applied to change this equilibrium state (in the absence of temperature change or soft elastic response). Residual, stress-free strain results from two-stage linking because the fraction $\xi$ of chains linked in the first stage are under tension and want to contract; the fraction $(1-\xi)$ linked in the second stage resists the contraction since it would lead to their extension in the plane perpendicular to the stretch direction, thereby raising their energy. We shall see the residual strains being almost the same fraction $(1-\xi)$ of the second stage linkage strain.

The deformation gradient $\underline{\underline{\lambda}}=\partial \mathbf{X} / \partial \mathbf{X}_{0}$ describes shape changes of the body, with $\mathbf{X}$ being the current position of a material point (in the target space) having been at $\mathbf{X}_{0}$ originally (in the reference space). For uniform distortions, the diagonal elements of $\underline{\underline{\lambda}}$ are simply the ratio of a current dimension to the corresponding original dimension. Thus the ratios of the current and original volumes is Det $[\underline{\underline{\lambda}}]$ which we must set equal to unity since rubber deformations are at constant volume. The off-diagonal elements are simple shears and will be illustrated as they are used. In the current smectic case, we assume the second stage linking is made in the smectic- $A$ state and that the deformation gradient $\underline{\underline{\lambda}}_{1}$ applied after the first step is a simple, volume- and layer-preserving shear:

$$
\underline{\underline{\lambda}}_{1}=\underline{\underline{\delta}}+\lambda_{1} \mathbf{m n}_{0} \rightarrow\left(\begin{array}{ccc}
1 & 0 & \lambda_{1} \\
0 & 1 & 0 \\
0 & 0 & 1
\end{array}\right) .
$$

In $\underline{\underline{\lambda}}_{1}$ above, $\underline{\underline{\delta}}$ is the unit tensor, $\mathbf{m}$ is the direction in the smectic planes of displacement in the shear, and $\mathbf{n}_{0}$ is the initial director which is along the layer normal since we start in the $\operatorname{Sm} A$ phase. The deformation gradient $\underline{\underline{\lambda}}_{1}$ at second stage cross-linking defines a second direction, $\mathbf{m}$, in the layer plane and thereby later gives $\mathrm{SmC}$ monodomains since tilt is in this selected direction. These stages of deformation are approximately the scheme of Hiraoka et al. [12] who applied simple shear to their partially cross-linked rubber which was likely in the $\operatorname{Sm} A$ state, but possibly entering the $\operatorname{SmC}$ state as solvent loss proceeded during second-stage linkage. When we take a particular coordinate system, $\mathbf{z}$ is along $\mathbf{n}_{0}$ and $\mathbf{x}$ is along $\mathbf{m}$ in-plane, in which case $\lambda_{1} \equiv \lambda_{x z}$. In analogy with the outcome of second-stage cross-linking of nematic elastomers under extensional strain, we can see how second-stage crosslinking under shear strain leaves behind a residual shear that is present even in the $\operatorname{Sm} A$ elastomer where there is no molecular tilt.

The elastic free-energy density of a nematic network can be simply generalized from that of classical rubber elasticity [2] to give

$$
f=\frac{1}{2} \mu \operatorname{Tr}\left[\underline{\underline{\ell}}_{0} \cdot \underline{\underline{\lambda}}^{\mathrm{T}} \cdot \underline{\underline{\ell}}_{\mathbf{n}}^{-1} \cdot \underline{\underline{\lambda}}\right],
$$

where $\mu$ is the shear modulus in the isotropic state and $\underline{\underline{\lambda}}$ is the deformation gradient suffered by the system since first stage linkage. The Gaussian distribution of chain shapes at formation and currently is characterized by their mean square dimensions. These are, respectively, proportional to their effective step length tensors (in an anisotropic distribution) $\ell_{0}$ and $\underline{\underline{\ell}}_{\mathbf{n}}$. The current step length tensor $\underline{\underline{\ell}}_{\mathbf{n}}$ takes account of 
any director rotation to the new $\mathbf{n}$ and any changes in order due to temperature change. When cross-linking is performed at more than one stage, the free-energy density in the nematic case is obtained by simple extension [18] of classical calculations on isotropic rubber of multistage linking and yields

$$
f=\frac{1}{2} \mu \operatorname{Tr}\left[\underline{\underline{A}} \cdot \underline{\underline{\lambda}}^{\mathrm{T}} \cdot \underline{\underline{\ell}}_{\mathbf{n}}^{-1} \cdot \underline{\underline{\lambda}}\right],
$$

where now $\underline{\underline{\lambda}}$ describes the total deformation since crosslinking was started and the composite second-rank tensor $\underline{\underline{A}}$ records the chemomechanical history during the crosslinking process. The shape tensors are

$$
\begin{aligned}
& \underline{\underline{\ell_{0}}}=(r-1) \mathbf{n}_{0} \mathbf{n}_{0}+\underline{\underline{\delta}}, \\
& \underline{\underline{\ell_{\mathbf{n}}}}=(r-1) \mathbf{n n}+\underline{\underline{\delta}}, \\
& \underline{\underline{A}}=\xi \underline{\underline{\ell_{0}}}+(1-\xi) \underline{\underline{\lambda}}_{1}^{-1} \cdot \underline{\ell}_{0} \cdot \underline{\underline{\lambda}}_{1}^{-T} \\
& \rightarrow\left(\begin{array}{ccc}
1+(1-\xi) r \lambda_{1}^{2} & 0 & -(1-\xi) r \lambda_{1} \\
0 & 1 & 0 \\
-(1-\xi) r \lambda_{1} & 0 & r
\end{array}\right) .
\end{aligned}
$$

The transpose of the inverse of a tensor is denoted by superscript $^{-T}$. All the tensors have had a factor of the effective step length perpendicular to the director taken out, leaving only the ratio $r$ of the parallel to the perpendicular step lengths, $\ell_{\|} / \ell_{\perp}$, that is a measure of the chain shape distribution's anisotropy. The $\underline{\underline{A}}$ tensor has been specialized to a fraction $\xi$ of links being formed initially when the shape distribution was characterized by the persistence tensor $\underline{\ell}_{0}$ and a fraction $1-\xi$ being formed after a deformation $\underline{\underline{\lambda}}_{1}$ was applied, the persistence tensor, $\ell_{0}$, not changing between the stages. The current step tensor, $\overline{\bar{\ell}}_{\mathbf{n}}$, in the $\operatorname{Tr}[\cdots]$ is also taken to depend on the same $r$. We are in effect assuming that these step lengths change little with temperature. The data of Hiraoka et al. [12], their Fig. 4 showing length change with temperature, suggests that chains elongate to a prolate shape distribution on cooling to the $\operatorname{Sm} A$ phase and that there is little shape change subsequently. This assumption makes for simplicity of analysis here, but can be straightforwardly removed if local nematic order were to change as well. For instance, a possible scenario would be $r>1$ in $\ell_{0}$ reflecting nematiclike order initially. If the second stage linkage were in an oblate $\operatorname{Sm} A$ state, the second $\ell_{0}$ in $\underline{\underline{A}}$ would be replaced by an $\ell_{1}$ with an anisotropy $r_{1}<\overline{1}$. The director $\mathbf{n}_{1}$ in $\ell_{1}$ would $\bar{b}$ e along the layer normal for $\operatorname{Sm} A$ second-stage lin $\overline{\bar{k}}$ age, or tilted when the linkage is in theSmC state. The generalization of Eq. (6) to many cross-linking stages with differing strains, and while changes in chain distribution occur, is also easily made [18].

Denote by $\underline{\underline{\lambda}}^{\mathrm{r}}$ the deformation connecting the initial state and relaxed state after completing all cross-linking and subsequent removal of imposed strain. Deformation with respect to this natural, relaxed shape will be denoted by $\underline{\underline{\lambda}}^{\prime}$ which means that overall:

$$
\underline{\underline{\lambda}}=\underline{\underline{\lambda}}^{\prime} \cdot \underline{\underline{\lambda}}^{\mathrm{r}}
$$

Returning $\underline{\underline{\lambda}}$ to Eq. (3), one obtains a free-energy density describing the initial relaxation and also subsequent shape changes:

$$
\begin{aligned}
f & =\frac{1}{2} \mu \operatorname{Tr}\left[\left(\underline{\underline{\lambda}}^{\mathrm{r}} \cdot \underline{\underline{A}} \cdot \underline{\underline{\lambda}}^{\mathrm{r}}\right) \cdot \underline{\underline{\lambda}}^{\prime \mathrm{T}} \cdot \underline{\underline{\ell}}_{\mathbf{n}}^{-1} \cdot \underline{\underline{\lambda}}^{\prime}\right] \\
& \equiv \frac{1}{2} \mu \operatorname{Tr}\left[\underline{\underline{\underline{\ell}}} \cdot \underline{\underline{\underline{\lambda}}}^{\mathrm{T}} \cdot \underline{\underline{\ell}}_{\mathbf{n}}^{-1} \cdot \underline{\underline{\lambda}}^{\prime}\right],
\end{aligned}
$$

where $\ell_{\mathrm{r}}=\underline{\underline{\lambda}}^{\mathrm{r}} \cdot \underline{\underline{A}} \cdot \underline{\underline{\underline{\lambda}}}^{\mathrm{rT}}$ plays the role of an effective step length tensor that encodes memory of the previous conditions of the sample. The relaxed state has $\underline{\underline{\lambda}}^{\prime}=\underline{\underline{\delta}}$. We find the relaxation, that occurs after the release of the constraints applied during cross-linking, by minimizing $f=\frac{1}{2} \mu \operatorname{Tr}\left[\underline{\underline{\lambda}}^{\mathrm{r}} \cdot \underline{\underline{A}} \cdot \underline{\underline{\lambda}}^{\mathrm{rT}} \cdot \ell_{\mathbf{n}}^{-1}\right]$ under the everpresent constraint of incompressibility, $\operatorname{Det}\left[\underline{\underline{\lambda}}^{\mathrm{r}}\right]=1$.

An obvious minimiser for this free energy would be [2] $\underline{\underline{\lambda}}^{\mathrm{r}}=\underline{\underline{\ell}}_{\mathbf{n}}^{1 / 2} \cdot \underline{\underline{W}} \cdot \underline{\underline{A}}^{-1 / 2} / \operatorname{Det}\left[\underline{\ell}_{\mathbf{n}}^{1 / 2} \cdot \underline{\underline{A}}^{-1 / 2}\right]^{1 / 3}$ which has been constructed to satisfy volume conservation, $\operatorname{Det}\left[\underline{\underline{\lambda}}^{\mathrm{r}}\right]=1$, and where $\underline{\underline{W}}$ is an arbitrary rotation. However, even with the extra freedom offered by $\underline{\underline{W}}$, such deformations do not preserve the layer spacing constraint arising from the convection of layers by deformations [15]:

$$
\begin{gathered}
d / d_{0}=1 /\left|\underline{\underline{\lambda}}^{-T} \cdot \mathbf{k}_{0}\right|=1, \\
\mathbf{k}=\underline{\underline{\lambda}}^{-T} \cdot \mathbf{k}_{0},
\end{gathered}
$$

where $\mathbf{k}_{0}$ is the initial (unit) direction of the layer normal in the solid, with $d_{0}$ the initial spacing, and $\mathbf{k}$ the final layer normal with $d$ the final spacing. [One may check that the shear imposed at second stage cross-linking, $\lambda_{1}$ in Eq. (1), is layer-preserving when layers have the normal $\mathbf{k}_{0}=\mathbf{n}_{0}=\mathbf{z}$.]

To respect the smectic phase, we accordingly restrict the initial relaxation $\underline{\underline{\lambda}}^{\mathrm{r}}$, the subsequent response $\underline{\underline{\lambda}}^{\prime}$, and thus also the overall response $\underline{\underline{\lambda}}$, to the volume- and layerpreserving forms:

$$
\begin{aligned}
& \underline{\underline{\lambda}}^{\mathrm{r}}=\left(\begin{array}{ccc}
\lambda_{\mathrm{xx}}^{\mathrm{r}} & 0 & \lambda_{\mathrm{xz}}^{\mathrm{r}} \\
0 & \frac{1}{\lambda_{\mathrm{xx}}^{\mathrm{r}}} & 0 \\
0 & 0 & 1
\end{array}\right) ; \\
& \underline{\underline{\lambda}}^{\mathrm{r}-\mathrm{T}}=\left(\begin{array}{ccc}
\frac{1}{\lambda_{\mathrm{xx}}^{\mathrm{r}}} & 0 & 0 \\
0 & \lambda_{\mathrm{xx}}^{\mathrm{r}} & 0 \\
-\frac{\lambda_{\mathrm{xz}}^{\mathrm{r}}}{\lambda_{\mathrm{xx}}^{\mathrm{r}}} & 0 & 1
\end{array}\right), \\
& \underline{\underline{\lambda}}^{\prime}=\left(\begin{array}{ccc}
\lambda_{\mathrm{xx}}^{\prime} & 0 & \lambda_{\mathrm{xz}}^{\prime} \\
0 & \frac{1}{\lambda_{\mathrm{xx}}^{\prime}} & 0 \\
0 & 0 & 1
\end{array}\right),
\end{aligned}
$$




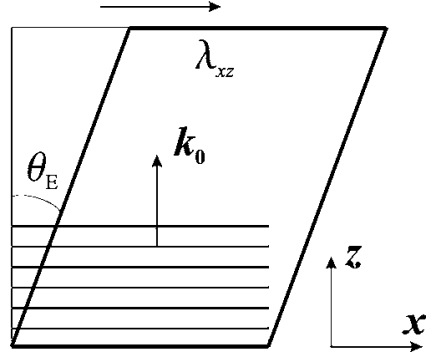

FIG. 1. Smectic $A$ elastomer with $\lambda_{x z}$ shear (bold shape) relative to the initial shape (faint). Note the absence of $\lambda_{z z}$ elongation and $\lambda_{z x}$ shear and hence the preservation of layer orientation and spacing.

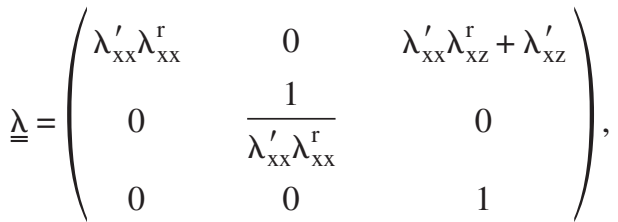

where the transpose of the inverse tensor, $\underline{\underline{\lambda}}^{\mathrm{r}-\mathrm{T}}$, is useful in finding the new layer normal after deformation, Eq. (11). One can see that this choice of the various $\underline{\underline{\lambda}}$ tensors leaves the direction and magnitude of the normal $\mathbf{z}$ unchanged when applied in Eq. (11).

\section{A. Relaxation after cross-linking}

A shear deformation $\underline{\underline{\lambda}}_{1}$, see Fig. 1, held during second stage cross-linking means that, on release, a sample does not return to its original shape, but retains a residual deformation $\underline{\underline{\lambda}}^{\mathrm{r}}$. Cross-links established in the sheared state ensure a memory of that shear. Shear deformation can be characterized by the angle $\theta_{\mathrm{E}}=\tan ^{-1} \lambda_{x z}$, in the notation of Hiraoka et al. [12], and is in general different from the director tilt angle $\theta$, see below. Inserting the relaxation (12) and the tensor $\underline{\underline{A}}$ (7) into the free-energy density (3), one obtains

$$
\begin{aligned}
f= & \frac{1}{2} \mu\left(\lambda_{x x}^{2}\left[1+(1-\xi) r \lambda_{1}^{2}\right]-\frac{1}{\lambda_{x x}^{2}}+r \lambda_{x z}^{2}\right. \\
& \left.-2 \lambda_{x x} \lambda_{x z}(1-\xi) r \lambda_{1}+1\right) .
\end{aligned}
$$

Minimization with respect to $\lambda_{x x}$ and $\lambda_{x z}$ yields

$$
\begin{aligned}
& \lambda_{x x}^{\mathrm{r}}=1 /\left(1+\gamma \lambda_{1}^{2}\right)^{1 / 4} ; \quad \lambda_{y y}^{\mathrm{r}}=\left(1+\gamma \lambda_{1}^{2}\right)^{1 / 4}, \\
& \lambda_{x z}^{\mathrm{r}}=(1-\xi) \lambda_{1} \lambda_{x x}^{\mathrm{r}}=(1-\xi) \lambda_{1} /\left(1+\gamma \lambda_{1}^{2}\right)^{1 / 4},
\end{aligned}
$$

where for brevity $\gamma$ denotes $r \xi(1-\xi)$.

There is an $x x$ contraction (not shown in Fig. 1) at second order in $\lambda_{1}$ : shears at constant volume can be viewed as extensions and contractions along diagonals that are perpendicular for small shears and become increasingly skewed on larger shear. For small shears there are equal extensions and contractions that are projected into the layer planes and nothing happens. For larger shears the extension diagonal is more nearly in the layer plane and thus on release the system tries to contract in the in-plane $(x)$ direction. The magnitude of contraction necessarily depends on $\lambda_{1}^{2}$ at lowest order since the contraction is independent of the sign of the shear $\lambda_{1}$. Given that the layer spacing is constant, $x x$ is the only possible contraction reaction, accompanied of course by a volume-preserving extension $\lambda_{y y}$.

The final relaxed shear $\lambda_{x z}^{\mathrm{r}}$ is less than that imposed at second linkage, $\lambda_{1}$, by virtue of this contraction and the reduced fraction of strands $(1-\xi)$ that resist the contraction.

Injecting the $\underline{\underline{\lambda}}^{\mathrm{r}}$ tensor into Eq. (9), we can now find the relaxed effective step length tensor $\ell_{\mathrm{r}}$ that records the full cross-linking and relaxation history:

$$
\stackrel{\ell_{\mathrm{r}}}{=}\left(\begin{array}{ccc}
1 / \lambda_{x x}^{\mathrm{r} 2} & 0 & 0 \\
0 & 1 / \lambda_{x x}^{\mathrm{r} 2} & 0 \\
0 & 0 & r
\end{array}\right) .
$$

This result is strange when seen from the perspective of nematic elastomers where relaxation of initial conditions generally gives a free energy for subsequent deformation of the form of Eq. (9) but with an effective step length and its own inverse appearing. Here, in the layer-constrained smectic system, the two tensors are not inverses of each other because full relaxation would have involved $z z$ and $z x$ which are forbidden by the layer rigidity.

\section{B. Spontaneous deformation in the $C$-phase}

Now we can calculate the deformation in response to lowering the temperature into the $\mathrm{SmC}$ phase and therefore developing a director tilt $\theta$ with respect to the layer normal, assumed constant along $\mathbf{z}$. We assume that the director is rigidly aligned to the layer normal in the $\operatorname{Sm} A$ phase. Evidence from $\operatorname{Sm} A$ experiments show that the smectic energy scale is about two orders of magnitude greater than the rubber scale and that this is a reasonable assumption. We make the further assumption that as tilt develops, the director is equally rigidly anchored to the equilibrium tilt angle and resists any mechanically inspired drive to deviate. This assumption can almost certainly not be made for de Vries $\operatorname{Sm} A$ phases where, for instance, molecular tilt and layer spacing are not simply related. Hiroaka et al. [12] use the notation $\theta_{\mathrm{x}}$ for the tilt angle. The superscript indicates it is the actual tilt between the layers and the director reflexes as seen by $\mathrm{x}$-ray scattering. Putting the deformation gradient with respect to the relaxed $\operatorname{Sm} A$ state, $\underline{\underline{\lambda}}^{\prime}$, into the free-energy density (9), one obtains

$$
\begin{aligned}
f= & \frac{1}{2} \mu\left(1+(r-1) \sin ^{2} \theta-2(r-1) \lambda_{x z}^{\prime} \sin \theta \cos \theta+\lambda_{x z}^{\prime 2}[r\right. \\
& \left.-(r-1) \sin ^{2} \theta\right]+\sqrt{1+\gamma \lambda_{1}^{2}}\left[\frac{\lambda_{x x}^{\prime 2}}{r}\left[r-(r-1) \sin ^{2} \theta\right]\right. \\
& \left.\left.+\frac{1}{\lambda_{x x}^{\prime 2}}\right]\right) .
\end{aligned}
$$

The components $\lambda_{x z}^{\prime}$ and $\lambda_{x x}^{\prime}$ are now decoupled. Minimizing over each of them gives

$$
\lambda_{x z}^{\prime}=(r-1) \sin \theta \cos \theta /\left[r-(r-1) \sin ^{2} \theta\right],
$$




$$
\lambda_{x x}^{\prime}=r^{1 / 4} /\left[r-(r-1) \sin ^{2} \theta\right]^{1 / 4}>1 .
$$

These further relaxations in response to director tilt are noticeably independent of the chemomechanical history given by the values of $\xi$ and $\lambda_{1}$. Returning these deformations to the free energy, one obtains the rubber elastic addition to the free energy on rotating the director:

$f=\frac{1}{2} \mu\left(\frac{r}{r-(r-1) \sin ^{2} \theta}+2 \frac{\sqrt{1+\gamma \lambda_{1}^{2}}}{r^{1 / 2}}\left[r-(r-1) \sin ^{2} \theta\right]^{1 / 2}\right)$.

This cost of rotation with respect to the energy of the relaxed, unrotated state, $f(\theta=0)=\frac{1}{2} \mu\left(1+\sqrt{1+\gamma \lambda_{1}^{2}}\right)$, for small $\theta$ takes the form

$$
f(\theta) \approx-\frac{1}{2} \mu \frac{r-1}{r}\left(\sqrt{1+\gamma \lambda_{1}^{2}}-1\right) \theta^{2} .
$$

We have presumed that the director is well-anchored to the equilibrium tilt angle of the $\mathrm{SmC}$ phase. Accordingly, this additional energy is a small perturbation tending to only weakly promote the $A$ to $C$ transition.

\section{The role of nonideality}

The nematic free energy used above is an ideal form in that, without layer constraints, it leads to soft elasticity. Particular models of compositional fluctuations and rigid rod cross-links can be shown to lead to nonideal, that is semisoft, nematic elasticity [2]. We now explore the effect suitable forms of nonideality might have on the spontaneous distortions following cross-linking under strain and later tilting of the director. We find the effects are minor, the anisotropy $r$ being enhanced additively by the degree of semisoftness, $\alpha$, and the previous results being essentially maintained. the reader not interested in these minor modifications can ignore this section, simply referring to the modified results (28) and (29) we derive for $\lambda_{x z}^{\prime}$ and $\lambda_{x x}^{\prime}$.

In general, if a nonideal addition is to be made to energies of the form of Eq. (3), then at second order in $\underline{\underline{\lambda}}$ it must be of the form $\frac{1}{2} \mu \alpha \operatorname{Tr}\left[\underline{\underline{B}} \cdot \underline{\underline{\lambda}}^{\mathrm{T}} \cdot \underline{\underline{C}} \cdot \underline{\underline{\lambda}}\right]$ where the tensor $\underline{\underline{B}} \neq \ell_{0}$ and $\underline{\underline{C}} \neq \underline{\underline{\ell}}_{\mathbf{n}}^{-1}$. Otherwise the $\alpha$ term can be subsumed into the first term by the induction of a spontaneous distortion. The degree of nonideality is set by the factor $\alpha$ and by the magnitudes of the tensors $\underline{\underline{B}}$ and $\underline{\underline{C}}$.

The form of the tensors $\underline{\underline{B}}$ and $\underline{\underline{C}}$ in the $\alpha$-addition to the free energy in the literature arising from the compositional fluctuations mechanism for semisoftness turns out not to couple to the layer-constrained relaxations of the present problem. Instead we adopt the addition $\frac{1}{2} \mu \alpha \operatorname{Tr}\left[\mathbf{n}_{0} \mathbf{n}_{0} \cdot \underline{\underline{\lambda}}^{\mathrm{T}} \cdot \underline{\underline{\delta}}_{\mathbf{n}}^{\mathrm{r}} \cdot \underline{\underline{\lambda}}\right]$ where $\underline{\underline{\delta}}_{\mathbf{n}}^{\mathrm{rr}}$ is a perpendicular projector that selects out the components $\mathbf{y y}+\mathbf{s s}$ in the plane perpendicular to the current director $\mathbf{n}$. This choice of $\underline{\underline{B}}$ and $\underline{\underline{C}}$ obeys the requirement for semisoft response given above. The yy element is not relevant to this part of the problem. The vector $\mathbf{s}=\cos \theta \mathbf{x}-\sin \theta \mathbf{z}$ is perpendicular to $\mathbf{n}$ and in the plane of $\mathbf{n}_{0}$ and $\mathbf{n}$. The multistage generalization of this particular nonideal addition to the free energy is

$$
f_{\alpha}=\frac{1}{2} \mu \alpha \operatorname{Tr}\left[\underline{\underline{B}} \cdot \underline{\underline{\lambda}}^{\mathrm{T}} \cdot \mathbf{s} \mathbf{s} \cdot \underline{\underline{\lambda}}\right] \text { with }
$$

$$
\begin{gathered}
\underline{\underline{B}}=\xi \mathbf{n}_{0} \mathbf{n}_{0}+(1-\xi) \underline{\underline{\lambda}}_{1}^{-1} \cdot \mathbf{n}_{0} \mathbf{n}_{0} \cdot \underline{\underline{\lambda}}_{1}^{-T} \\
\rightarrow\left(\begin{array}{ccc}
(1-\xi) \lambda_{1}^{2} & 0 & -(1-\xi) \lambda_{1} \\
0 & 0 & 0 \\
-(1-\xi) \lambda_{1} & 0 & 1
\end{array}\right) .
\end{gathered}
$$

Taking relaxation of the form (12) and minimizing the ideal free energy plus the nonideal addition with respect to $\lambda_{x z}$ and $\lambda_{x x}$ as before, one obtains simple modifications of Eq. (17) and (16):

$$
\begin{gathered}
\lambda_{x x}^{\mathrm{r}}=1 /\left(1+\gamma^{\prime} \lambda_{1}^{2}\right)^{1 / 4}, \\
\lambda_{x z}^{\mathrm{r}}=(1-\xi) \lambda_{1} \lambda_{x x}^{\mathrm{r}}=(1-\xi) \lambda_{1} /\left(1+\gamma^{\prime} \lambda_{1}^{2}\right)^{1 / 4},
\end{gathered}
$$

where now $\gamma^{\prime}=(r+\alpha) \xi(1-\xi)$. This is the relaxation of a nonideal smectic- $A$ elastomer to its stress-free state after the strain $\lambda_{1}$ imposed during second cross-linking has been released.

Armed with $\underline{\underline{\lambda}}^{\mathrm{r}}$ and splitting the total deformation up as in Eq. (8), one rewrites the nonideal term as one did the ideal term in Eq. (9) to get $\underline{\underline{r}}_{\mathrm{r}}$ with now

$$
\underline{\underline{B}}_{\mathrm{r}}=\underline{\underline{\lambda}}^{\mathrm{r}} \cdot \underline{\underline{B}} \cdot \underline{\underline{\lambda}}^{\mathrm{r} \mathrm{T}} \equiv\left(\begin{array}{ccc}
\frac{\xi(1-\xi) \lambda_{1}^{2}}{\left(1+\gamma^{\prime} \lambda_{1}^{2}\right)^{1 / 2}} & 0 & 0 \\
0 & 0 & 0 \\
0 & 0 & 1
\end{array}\right)
$$

being an effective step length tensor that encodes into the nonideality a memory of the previous conditions of the sample. One now minimizes over the components of the subsequent deformation $\underline{\underline{\lambda}}^{\prime}$ and obtains the equivalent of Eqs. (20) and (21):

$$
\begin{gathered}
\lambda_{x z}^{\prime}=\frac{(r+\alpha-1) \sin \theta \cos \theta}{r+\alpha-(r+\alpha-1) \sin ^{2} \theta}, \\
\lambda_{x x}^{\prime}=r^{1 / 4} /\left[r-\left(r-\frac{1+\gamma \lambda_{1}^{2}}{1+\gamma^{\prime} \lambda_{1}^{2}}\right) \sin ^{2} \theta\right]^{1 / 4} .
\end{gathered}
$$

The $\lambda_{x z}^{\prime}$ relaxation in response to the director tilt remains independent of the chemo-mechanical history, even when nonideality is introduced, while $\lambda_{x x}^{\prime}$ becomes weakly dependent. Shear is maximal at a molecular tilt $\theta_{\max }$ $=\tan ^{-1}(\sqrt{r+\alpha})$ where it is $\lambda_{x z}^{\prime}(\max )=(r+\alpha-1) /(2 \sqrt{r+\alpha})$. As tilt gets very large, shear recedes and is replaced by in-plane elongation $\lambda_{x x}^{\prime} \rightarrow\left[r\left(1+\gamma^{\prime} \lambda_{1}^{2}\right) /\left(1+\gamma \lambda_{1}^{2}\right)\right]^{1 / 4}$ in the limit of large tilts.

\section{REPRESENTATIVE NUMERICAL RESULTS}

We present here the relaxation on completion of crosslinking and also the relaxation that occurs on increasing tilt on cooling in the $\mathrm{SmC}$ phase. We take representative values of the anisotropy, $r$, in the region of $r=2.4$ as deduced from the thermal elongations observed by Hiraoka et al. [12] on cooling from the isotropic to the nematic/smectic- $A$ state. The division of cross-links $\xi$ and $1-\xi$ between the first and 


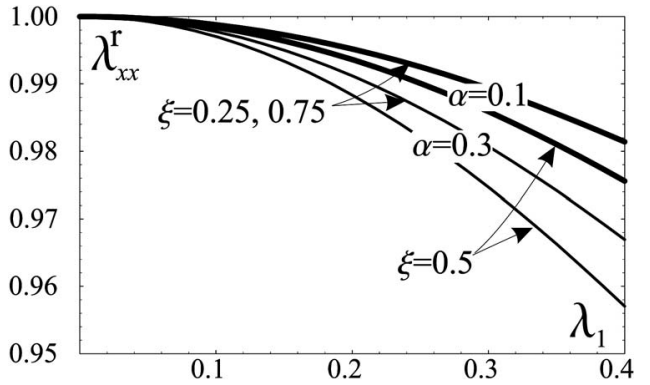

FIG. 2. Relaxational contraction $\lambda_{x x}^{\mathrm{r}}$ of a smectic- $A$ elastomer after a shear $\lambda_{1}$ was imposed during the second stage of crosslinking. Elastomers are shown with fractions $\xi=0.25,0.5$, and 0.75 of their links formed at first stage linkage and with either nonideality $\alpha=0.1$ and anisotropy $r=2.5$ (heavy lines) or with $\alpha=0.3$ and $r=4.5$ (light lines).

second cross-linking phases is less certain; we illustrate with a range of values. The degree of semisoftness is also uncertain, but it simply adds to the value of $r$-see the parallel between the ideal results (16) and (17), and the nonideal results (25) and (26) for relaxation after linkage, and the corresponding pairs of results for tilt-induced relaxation (20), (21), (28), and (29). We thus examine a range of $r$ values to accommodate this uncertainty.

Figure 2 shows the contraction $\lambda_{x x}^{\mathrm{r}}$ after the second stage of cross-linking in the $\operatorname{Sm} A$ phase as a function of the imposed simple shear $\lambda_{1}$, for the fractions $\xi=0.25,0.5$, and 0.75 of first stage linkage. The relaxation is modest even up to the large imposed second-stage shears deformations explored. The response for $\xi$ is equal to that for the case $1-\xi$, as consultation of Eq. (16) (ideal case) and Eq. (25) (nonideal case) confirms. Figure 3 shows the residual shear $\lambda_{x z}^{\mathrm{r}}$ remaining after the second stage linkage. Nonideality makes a hardly perceptible difference to $\lambda_{x z}^{\mathrm{r}}$ and is denoted, as in Fig. 2, by heavy and light lines, labeled only in the case $\xi$ $=0.25$. Since the contraction is so small, that is $\lambda_{x x}^{\mathrm{r}} \simeq 1$, then the residual shear is closely related to the imposed shear by the fraction of second-stage links: $\lambda_{x z}^{\mathrm{r}} \simeq(1-\xi) \lambda_{1}$ as expected and as can be confirmed from Eqs. (17) and (26).

The in-plane elongation induced by the tilt that grows on cooling into the $\mathrm{SmC}$ phase is shown in Fig. 4 against tilt. Both the systems $r=2.5$ and $r=4.5$ have suffered the same shear, $\lambda_{1}=0.3$ at second stage linking, and both have a nonideality of $\alpha=0.3$ and a first linkage fraction $\xi=0.5$. The

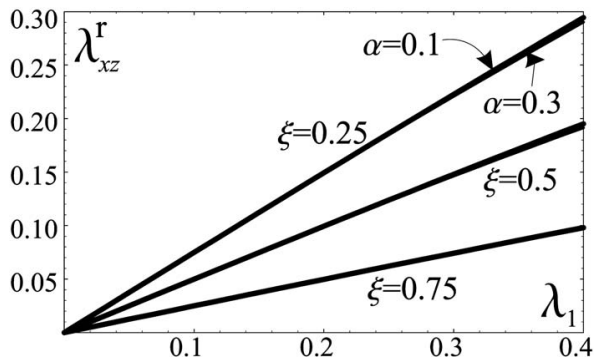

FIG. 3. Residual shear $\lambda_{x z}^{\mathrm{r}}$ of a smectic- $A$ elastomer after a shear $\lambda_{1}$ was imposed during the second stage of cross-linking. Conditions as in Fig. 2.

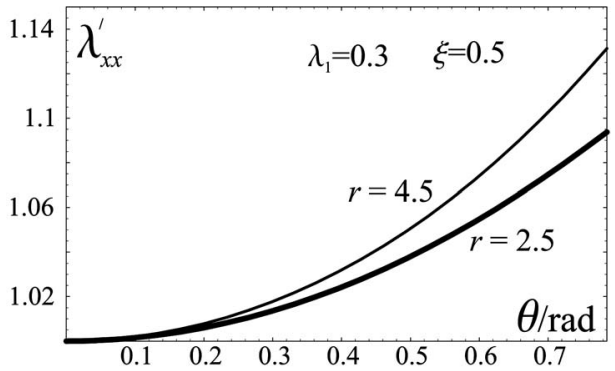

FIG. 4. In-plane elongation $\lambda_{x x}^{\prime}$ of a smectic $C$ elastomer as the director tilt $\theta$ develops on cooling.

corresponding simple shear induced by the director tilt is shown in Fig. 5. It has been most graphically observed by Hiraoka et al. [12]. We predict that shear reaches a maximum as tilt increases. The maxima for the parameters of the figure should be at $\theta=1.03$ and 1.14 rad for $r=2.5$ and 4.5 , respectively. However we display tilt only up to $\theta=\pi / 4$.

Another way to display results is to record the angle of shear distortion, $\theta_{E}$, accumulated by a sample after relaxation on second linkage and then further as tilt proceeds, see Fig. 1 and the remarks there about the representation by Hiraoka et al. [12] of their data. Since $\theta_{\mathrm{E}}=\arctan \lambda_{x z}$, one requires the total simple shear, that is the $x z$ element of Eq. (8), to get the angle

$$
\theta_{\mathrm{E}}=\arctan \left[\left(\underline{\underline{\lambda}}^{\prime} \cdot \underline{\underline{\lambda}}^{\mathrm{r}}\right)_{x z}\right]=\arctan \left[\lambda_{x x}^{\prime} \lambda_{x z}^{\mathrm{r}}+\lambda_{x z}^{\prime}\right] .
$$

We display the shear angle against director tilt in Fig. 6 where the off-set at $\theta=0$ measures the residual shear $\lambda_{x z}^{\mathrm{r}}$ remaining after cross-linking but still in the $\operatorname{Sm} A$ phase.

\section{SUMMARY AND INTERPRETATION}

We have modeled the two-step cross-linking of smectic elastomers with shear applied before, and held during, the second stage. The essence of this problem is of spontaneous shear distortions arising from the redirection of uniaxial directional order, rather than elongation or contraction arising from its change of magnitude. For simplicity we have assumed that the underlying prolate, nematic order, and thus the elongation of chains holds in the $\operatorname{Sm} A$ second stage linkage. We further assume the order remains largely invariant on cooling deeper into the $\mathrm{SmC}$ phase, and hence as tilt

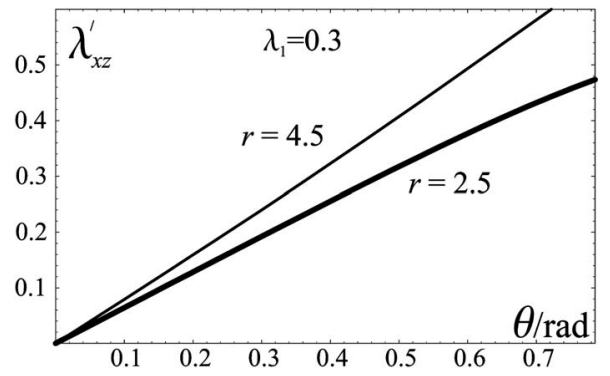

FIG. 5. Simple shear $\lambda_{x z}^{\prime}$ of a smectic $C$ elastomer as the director tilt $\theta$ develops on cooling. It is independent of the first stage linkage fraction $\xi$. 


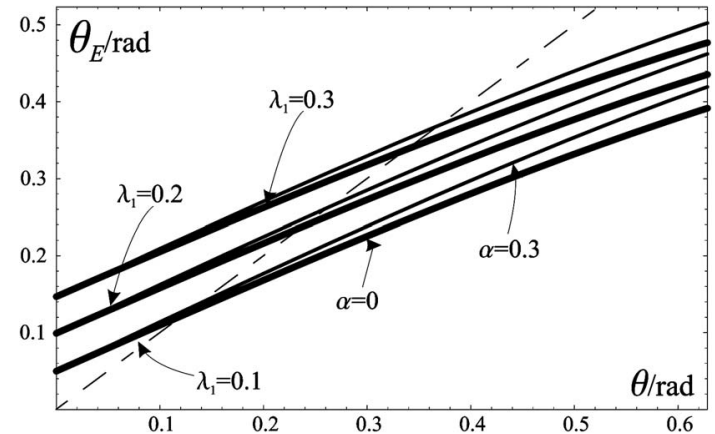

FIG. 6. Shear angle $\theta_{\mathrm{E}}$ of smectic elastomers as the director tilt $\theta$ develops on cooling. Each pair of curves shares the same crosslinking shear $\lambda_{1}$ and differ in their nonideality; $\alpha=0$ heavy line, $\alpha=0.3$ light line. The dashed line $\theta_{\mathrm{E}}=\theta$ emphasizes how mechanical and molecular tilt diverge from each other as a result of twostage cross-linking. ( $\xi=0.5$ in all cases.)

proceeds. Oblate chains $(r<1)$ are probably more realistic in the $\operatorname{Sm} A$ and $\operatorname{Sm} C$ states. Calculation yields negative shears with respect to the relaxed $\operatorname{Sm} A$ state if instead an oblate smectic state is adopted on second linkage and then rotated on entering the $\mathrm{SmC}$ phase. Such shear is not observed experimentally. $\operatorname{Sm} A$ oblate chain shapes arise from the constraints of layers with their normals along the director. It is plausible that the $\mathrm{SmC}$ chain shape tensor is modified by the director tilting away from the layer normal. Calculation of this modification and the negative shear effect of tilting oblate chains shows a resultant initial delay in spontaneous shear away from the residual shear in the $\operatorname{Sm} A$ state as tilt proceeds. We return to such complex connections between director tilt and spontaneous shear when a definitive determination of the state $(A$ or $C$ ) pertaining during linkage is available.

Of the shear imposed at the second stage of linkage, the fraction of shear retained after relaxation in the free state closely follows the fraction of cross-links formed in the second stage. The shear then increases further as director tilt is spontaneously developed. The $\mathrm{SmC}$ phase is taken to be untwisted in order that a unique, biaxial shape change develops macroscopically. The effect we describe in this large amplitude theory generalizes the continuum model of such mechanoclinic effects first proposed many years ago [7]. When shears are large, nonlinear elastic theories are essential. One can adopt Lagrangian elasticity models as in [5] or a molecular-based model such as the one we have presented here.

Recent experiments on the spontaneous distortion associated with tilt in $\mathrm{SmC}$ elastomers [12] report effective shear angles $\theta_{E}$ varying with tilt $\theta$ qualitatively like that plotted in Fig. 6, the agreement on heating being better than on cooling. The authors report residual strain above the $A C$ transition. Below the transition there is a small region of no increase of shear from the residual value even though the molecular tilt, as evidenced from x-ray scattering, is increasing. Their other measures of tilt, for instance, from sample dimension (and hence layer spacing) change, behave somewhat differently. To account for all details of tilt, it is possible that one must consider the response of a de Vries phase and also the oblate effects mentioned above.

This work shows that it is not necessary to invoke any special orientational ordering properties of the cross-links to explain the experimental result (corresponding to Fig. 6) of mechanical tilt, that is $\theta_{E} \neq 0$, where there is clearly no molecular tilt at all, $\theta=0\left(\theta_{X}=0\right.$ in the notation of Hiraoka et al. [12]). The residual shear $\lambda_{x z}^{\mathrm{r}}$ existing before the onset of molecular tilt can be explained by the simple, classical ideas of multistage cross-linking. Such linkage induces a mixture of chain tensions (in the fraction $\xi$ of chains) and compressions (in the fraction $1-\xi$ ) and a residual strain results such that there is zero overall stress in the relaxed, strained state.

When mesogen tilt starts, it is equally problematic to invoke the special orientation features of cross-links then being diluted by the tilting majority. If this were the case, one would eventually have the identification of $\theta_{E}$ with $\theta_{X}$ and the simple picture of mechanical tilt of the sample following director tilt. Again, Fig. 6 and its experimental equivalent [12] rule this out: the slope $d \theta_{E} / d \theta_{X}$ tends to values $\lesssim 1 / 2$ for large tilt. To emphasize the difficulty with invoking mechanisms leading to the identification of the mechanical and molecular tilt angles, the line $\theta_{E}=\theta_{X}$ has been dotted in Fig. 6. Clearly it is only at the accidental point where the lines $\theta_{E}\left(\theta_{X}\right)$ and $\theta_{E}=\theta_{X}$ cross that one can take such a view.

We are grateful to E.M. Terentjev for critically reading this paper and to the EPSRC for support of J.M.A. We thank Hiraoka et al. for clarifying the experimental situation to us and for a preprint of their results.
[1] L. Golubovic and T. C. Lubensky, Phys. Rev. Lett. 63, 1082 (1989).

[2] M. Warner and E. Terentjev, Liquid Crystal Elastomers (Oxford University Press, Oxford, 2003).

[3] I. Kundler and H. Finkelmann, Macromol. Rapid Commun. 16, 679 (1995).

[4] H. Finkelmann, I. Kundler, E. Terentjev, and M. Warner, J. Phys. II 7, 1059 (1997).

[5] O. Stenull and T. C. Lubensky, Phys. Rev. Lett. 94, 018304 (2005).

[6] J. M. Adams and M. Warner, Phys. Rev. E 72, 011703 (2005).
[7] E. M. Terentjev and M. Warner, J. Phys. II 4, 849 (1994).

[8] M. Brehmer, A. Wiesemann, R. Zentel, K. Siemensmeyer, and G. Wagenblast, Polym. Prepr. (Am. Chem. Soc. Div. Polym. Chem.) 34, 708 (1993).

[9] M. Brehmer, A. Wiesemann, R. Zentel, K. Siemensmeyer, and G. Wagenblast, Macromol. Chem. Phys. 195, 1891 (1994).

[10] I. Benne, K. Semmler, and H. Finkelmann, Macromol. Rapid Commun. 15, 295 (1994).

[11] K. Hiraoka and H. Finkelmann, Macromol. Rapid Commun. 22, 456 (2001).

[12] K. Hiraoka, W. Sagano, T. Nose, and H. Finkelmann, 
Macromolecules 38, 7352 (2005).

[13] E. Nishikawa and H. Finkelmann, Macromol. Chem. Phys. 200, 312 (1999).

[14] T. C. Lubensky, E. M. Terentjev, and M. Warner, J. Phys. II 4, 1457 (1994).

[15] J. M. Adams and M. Warner, Phys. Rev. E 71, 021708 (2005).
[16] M. J. Osborne and E. M. Terentjev, Phys. Rev. E 62, 5101 (2000).

[17] J. Kupfer and H. Finkelmann, Makromol. Chem., Rapid Commun. 12, 717 (1991).

[18] G. Verwey and M. Warner, Macromolecules 28, 4299 (1995). 